\title{
Das neue Bestäuberbeet im Botanischen Garten Frankfurt
}

\author{
Hilke Steinecke, Andreas König \& Thomas Moos
}

\begin{abstract}
Abtract
A small pollination bed was created in the Botanical Garden Frankfurt in 2020. There is also information given on various pollinator syndromes. In addition, information on pollination biology aspects is shown on selected plants in the garden. The area around the pollination bed is furnished with seating and can be used by groups as a green classroom.
\end{abstract}

\section{Zusammenfassung}

Im Botanischen Garten wurde im Jahr 2020 ein kleines Bestäuberbeet angelegt. Dazu gibt es Informationen zu verschiedenen Bestäubersyndromen. Ergänzend werden an ausgewählten Pflanzen im Garten Hinweise zu bestäubungsbiologischen Aspekten gegeben. Der Platz um das Bestäuberbeet ist mit Sitzgelegenheiten ausgestattet und kann von Gruppen als Grünes Klassenzimmer genutzt werden.

\section{Einleitung}

Im Fußball heißt es „Das Runde muss ins Eckige“, bei den Pflanzen dagegen „Der Pollen muss auf die Narbe“. Zugegeben, dieser flache Fußballwitz wird der Komplexität des Lebens nicht gerecht, aber hinter beiden Fällen steckt ein Erfolgsprinzip mit Siegern und Verlierern. In der seit Jahrmillionen andauernden Koevolution von Blütenpflanzen und ihren Bestäubern haben sich unglaubliche Spezialisierungen entwickelt, die hier nicht einmal andeutungsweise angerissen werden können. Wir möchten nur als Beispiele dazu auf den Entdecker der Blütenbestäubung, Christian KonRAd Sprengel (1750 - 1816), sowie die schöne Artikelreihe von Weber, Gerlach et al. in dieser Zeitschrift (siehe Literaturliste am Ende des Beitrages) hinweisen.

Die Blütenökologie, also die Wechselwirkungen zwischen Blütenpflanzen und ihren Bestäubern, steht als gemeinsames Leitthema im Fokus von Palmengarten und Botanischem Garten. Als herausragende Lernorte für dieses Thema bieten unsere beiden Gärten Raum für viele Aktivitäten aus dem Themenbereich Pflanzen, Blüten, Bestäubung und Insekten. Zu nennen wären zum Beispiel unsere Angebote der Grünen Schule und die Bildungseinrichtung „Kinder im Garten“ im Palmengarten sowie unser Foto-Dokumentationsprojekt im Internet auf der Bürgerwissenschaftsplattform iNaturalist (https://www.inaturalist.org/projects/tiere-undpilze-in-frankfurts-botanischen-garten).
Ein ganz besonderes Highlight ist natürlich das neue Blüten- und Schmetterlingshaus im Palmengarten inklusive einer Dauerausstellung zur Blütenökologie passend zum 150-jährigen Palmengartenjubiläum (vgl. Steinecke et al. 2021 in dieser Ausgabe). Draußen im Garten können dann als Ergänzung zu den tropischen Schmetterlingen und ihren Nektarpflanzen die in Frankfurt frei lebenden Arten und ihre Beziehungen zueinander beobachtet werden.

\section{Blüten und ihre Bestäuber im Botanischen Garten}

Als Ergänzung zum Blüten- und Schmetterlingshaus wurde im Botanischen Garten im Jahr 2020 ein kleines Bestäuberbeet angelegt, welches sich im südlichen Bereich des Botanischen Gartens zwischen dem Senckenbergischen Arzneipflanzengarten und der Wasserpflanzensammlung befindet. Die Planung erfolgte nach dem Motto „Nektar, Duft und Farbe " und wurde mit Materialien und Tipps aus anderen Botanischen Gärten und schulbiologischen Einrichtungen unterstützt

Vor dem Bestäuberbeet sind halbschattige Sitzgelegenheiten unter alten Tulpenbäumen (Liriodendron tulipifera) und einer Persischen Eiche (Quercus macranthera) mit zwei langen Holzbänken auf Mauern vorhanden. Der kleine Platz kann von Schulklassen oder Kindergruppen als Grünes Klassenzimmer genutzt werden, um Blütenbesucher zu beobachten und Lehrinhalte zu vermitteln. 


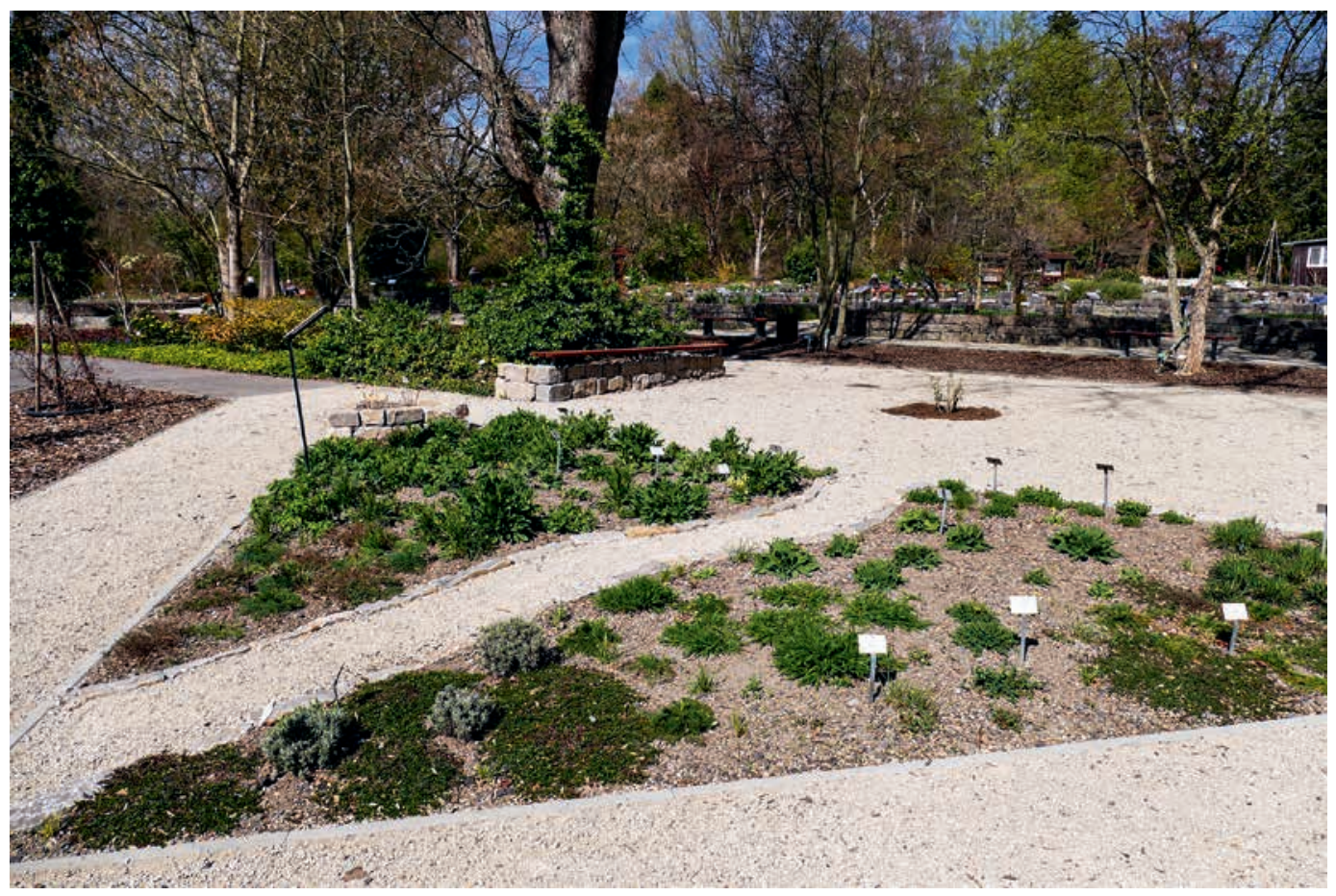

Abb. 1: Übersicht über das Bestäuberbeet im Frühlingsaspekt mit gerade frisch austreibenden Pflanzen. (Foto: H. STEINEcke)

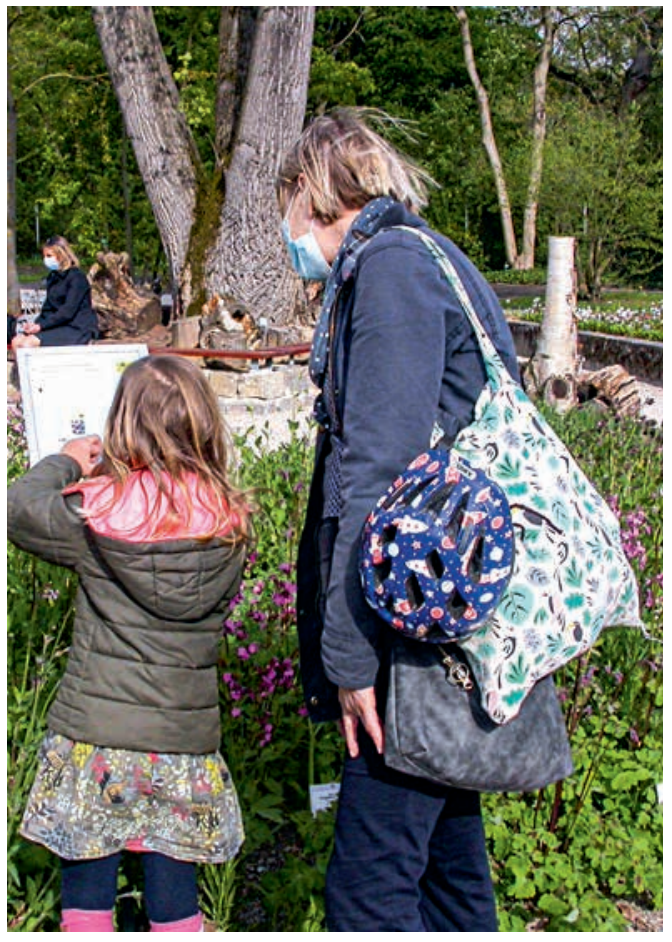

Abb. 2: Für Interessierte gibt es hier einiges zu erfahren.

(Foto: E. BRUde)

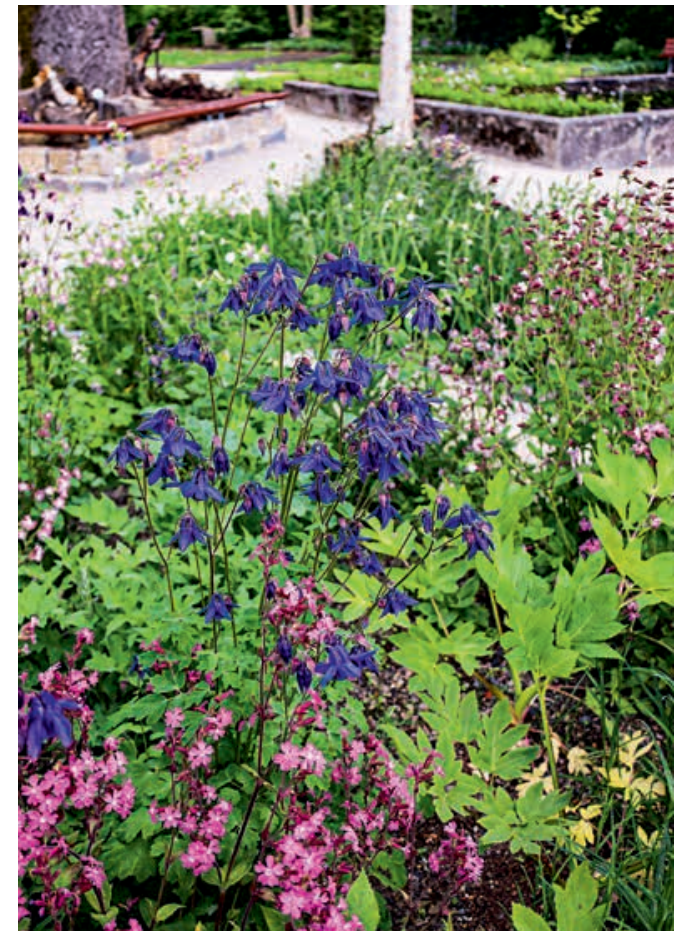

Abb. 3: Blühaspekt mit Lichtnelken und Akeleien im Mai.

(Foto: H. STEINECKe) 


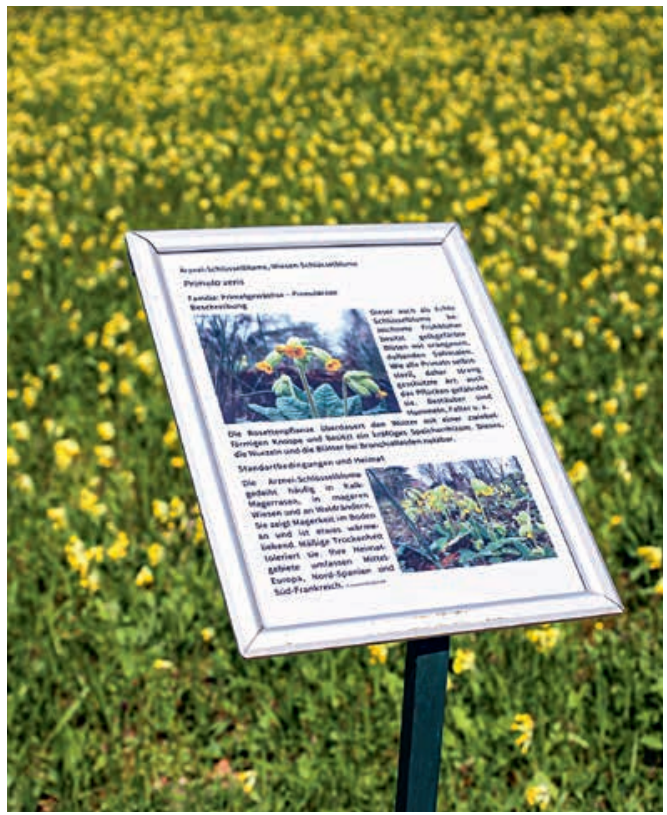

Abb. 4: Zu ausgewählten blütenbiologisch interessanten Pflanzen findet man im Garten räumlich und zeitlich verteilt allgemeine und speziell blütenbiologische Informationen. (Foto: H. STEINECKe)

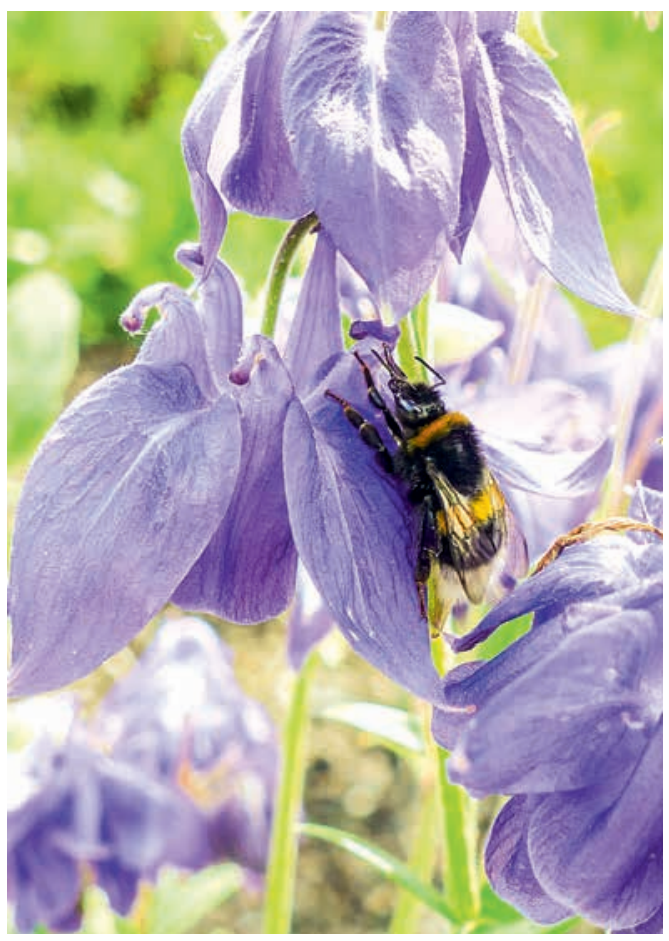

Abb. 5: Eine Hummel beißt den Nektarsporn einer Akelei auf und betätigt sich als Nektardieb. (Foto: H. Steinecke)
Auf der relativ kleinen Fläche des Bestäuberbeetes kann natürlich nur eine entsprechend kleine Auswahl von etwa 20 bestäubungsökologisch interessanten Arten zusammen mit drei Informationstafeln zu Bestäubersyndromen gezeigt werden. Wichtig war uns auch eine insektengerechte Umgebung mit Totholz, Stein-, Lehm- und Sandhaufen, um die Lockwirkung zu erhöhen.

Darüber hinaus wurden etwa 100 Tafeln vorbereitet, die, je nach Blütenangebot zeitlich und räumlich verteilt, Informationen im rund sechs Hektar großen Garten bereithalten. Aufgrund der pflanzensoziologischen Gestaltung großer Teile des Botanischen Gartens kann in den biotopartigen Anlagen mit zahlreichen Kleinlebensräumen die Vielfalt von Bestäubungsmechanismen hautnah und live erlebt werden.

Die Infotexte für diese Pflanzenstationen sind bewusst kurzgehalten und beschränken sich auf jeweils fünf Sätze plus Abbildungen. Nachfolgend soll eine kleine Auswahl von Pflanzen mit unterschiedlichen bestäubungsökologischen Aspekten vorgestellt werden.

\section{Auswahl kurzer blütenbiologischer Infos zu verschiedenen Arten} Aquilegia vulgaris (Gemeine Akelei, Ranunculaceae, Blumentyp: Glockenblume)

Die Blüten haben fünf gleichartige Blütenblätter sowie fünf gespornte Nektarblätter. Manche Hummeln, aber nicht die Bienen, haben ausreichend lange Rüssel, um den Nektar vom Grund der Sporne aufzusaugen. Gelegentlich sind sie aber „faul“ (oder kurzrüsselig) und beißen den Sporn einfach auf, um leichter an den Nektar zu kommen. Bei diesem Nektarraub kommen sie weder an den Staubblättern noch an den Narben vorbei und sind dann auch keine Bestäuber. In der Akeleiblüte reift der Pollen, bevor die Narben empfängnisbereit sind. Diese Vormännlichkeit verhindert Selbstbestäubung.

Buddleja davidii (Schmetterlingsstrauch, Scrophulariaceae, Blumentyp: Trichterblume)

Die zwittrigen, nach Honig duftenden Blüten der aus Tibet und dem zentralen und östlichen China 


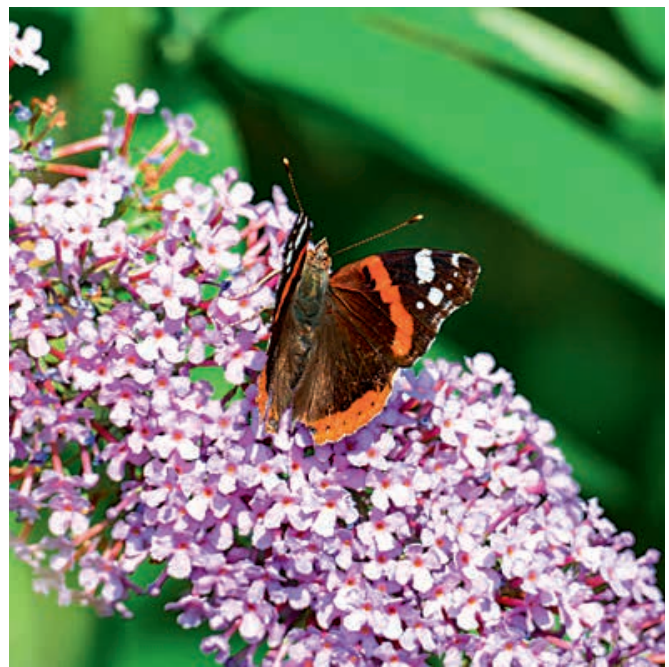

Abb. 6: Admiral (Vanessa atalanta) auf einem Schmetterlingsstrauch. (Foto: H. STEINECKe)

stammenden Art sind vierzählig und bilden einen endständigen, schmalkegeligen Blütenstand. Die lilafarbenen Blütenkronblätter sind zu einer etwa einem Zentimeter langen, engen Kronröhre verwachsen und besitzen ausgebreitete Kronzipfel. Sie sind im Bereich des Röhreneingangs kräftig gelb gefärbt; bei der Wildform ist die restliche Blüte blaulila, es gibt aber auch weiße, rosafarbene, rote und purpurfarbene Kulturformen. Die Narben und Staubbeutel bleiben in der engen Kronröhre verborgen. Die Bestäubung erfolgt durch langrüsselige Insekten, insbesondere durch Tagfalter, Taubenschwänzchen, Honigbienen, Hummeln und Holzbienen. Der Schmetterlingsstrauch blüht in einer bei uns relativ blütenarmen Zeit im Juli und August und ist durch sein reiches Nektarangebot immer ein Magnet für Insekten.

\section{Cytisus scoparius (Besenginster, Fabaceae,} Blumentyp: Schmetterlingsblume)

Die Blütenkrone ist in Fahne, Flügel und Schiffchen gegliedert. Das Schiffchen ist mit den seitlichen beiden Flügeln verzahnt und verbirgt den gespannten langen Griffel sowie die Staubblätter. Setzt sich eine schwere Biene oder Hummel auf das Schiffchen, drückt es dieses nach unten, um an den Nektar zu gelangen. Dadurch lösen sich die Blütenflügel, das Schiffchen öffnet sich und Griffel sowie Staubblätter schnellen explosionsartig her-

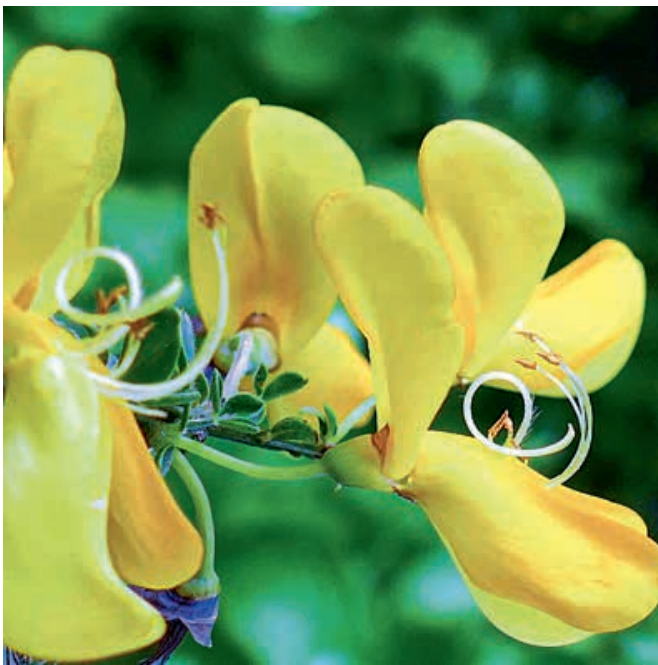

Abb. 7: An dieser Ginster-Blüte wurde bereits der Explosionsmechanismus ausgelöst. (Foto: H. STEINeCKe)

aus: Zuerst die fünf kurzen, danach die fünf langen Staubblätter, so dass der Bestäuber erst am Bauch und dann am Rücken mit Pollen eingepudert wird.

Dianthus superbus (Prachtnelke, Caryophyllaceae, Blumentyp: Stieltellerblume)

Die Blüten der Prachtnelke haben eine lange enge Kelchröhre, von der im rechten Winkel dazu die rosa bis weißen Blütenblätter abstehen. Das schmale Stielchen heißt Nagel, der flächige Ab-



Abb. 8: Ein Taubenschwänzchen (Macroglossum stellatarum) saugt im Schwirrflug den Nektar aus einer Blüte der Prachtnelke. (Foto: H. STEInecke) 
schnitt Platte. Die Fransen an den Kronblättern üben auf Insekten eine große Anziehungskraft aus. Die Blüten verströmen einen starken, vanilleähnlichen Duft. Am Grunde der Röhre wird Nektar abgegeben. Den können nur Schmetterlinge mit ihrem langen Rüssel aufnehmen, entweder auf der Platte sitzend oder im Schwirrflug wie das Taubenschwänzchen.

\section{Epipactis helleborine (Breitblättrige Sumpf- wurz, Orchidaceae, Blumentyp: Lippenblume)} Das untere Kronblatt von Orchideen ist oft zungenartig verlängert, dient Insekten als Landeplatz und wird als Lippe (Labellum) bezeichnet. Diese Orchidee bildet im hinteren Teil der Lippe Nektar, der hier nicht wie bei vielen anderen Orchideen in einem Sporn verborgen ist. Orchideen setzen bei der Bestäubung alles auf eine Karte, denn die Pollenkörner werden nicht einzeln als Blütenstaub ausgebreitet, sondern in Form von Pollenpaketen den Bestäubern auf einer bestimmten Stelle aufgeladen. Die zwei Pollenpakete (Pollinien) der Blüte sind über zwei Stielchen mit einer Klebscheibe verbunden, die gesamte Einheit heißt Pollinarium.

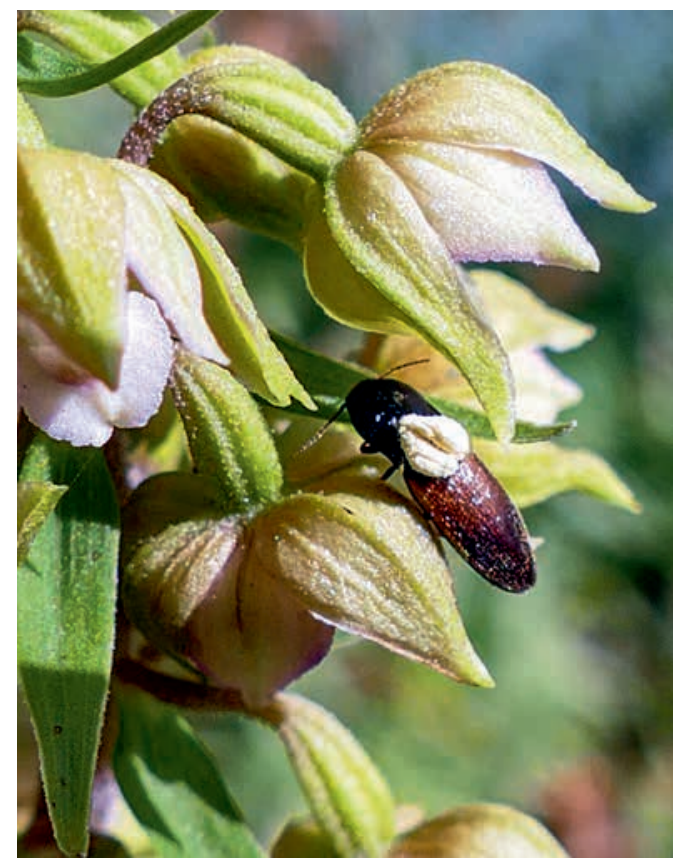

Abb. 9: Dieser kleine Schnellkäfer wurde während des Blütenbesuches der Breitblättrigen Sumpfwurz mit Pollenpaketen beladen. (Foto: H. STEINECKe)

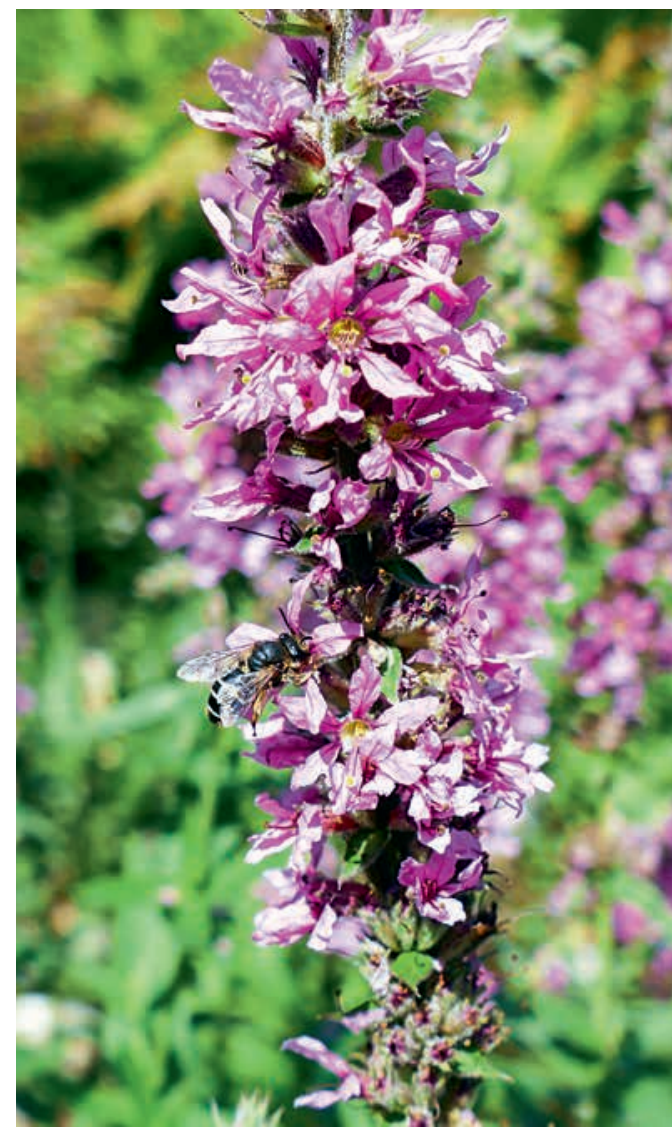

Abb. 10: Blutweiderich mit Blutweiderich-Sägehornbiene (Melitta nigricans). (Foto: H. STEINeCKe)

Krabbelt das Insekt in die Blüte, bleibt das Pollinarium meist im Bereich des Kopfes oder Rückens kleben. Es wird bei einem folgenden Blütenbesuch auf die Narbe übertragen.

\section{Lythrum salicaria (Blutweiderich, Lythraceae, Blumentyp: Trichterblume)}

Blutweiderich hat drei verschiedene Blütentypen. Langgriffelige mit kurzen und mittellangen Staubblättern, mittelgriffelige mit kurzen und langen Staubblättern, kurzgriffelige mit mittellangen und langen Staubblättern. Es kann nur Pollen anderer Blütentypen auf die Narbe übertragen werden, wodurch Fremdbestäubung gefördert wird. Die Blüten werden oft von Schmetterlingen aufgesucht, aber auch von der auf den Blutweiderich spezialisierten Blutweiderich-Sägehornbiene (Melitta nigricans). 


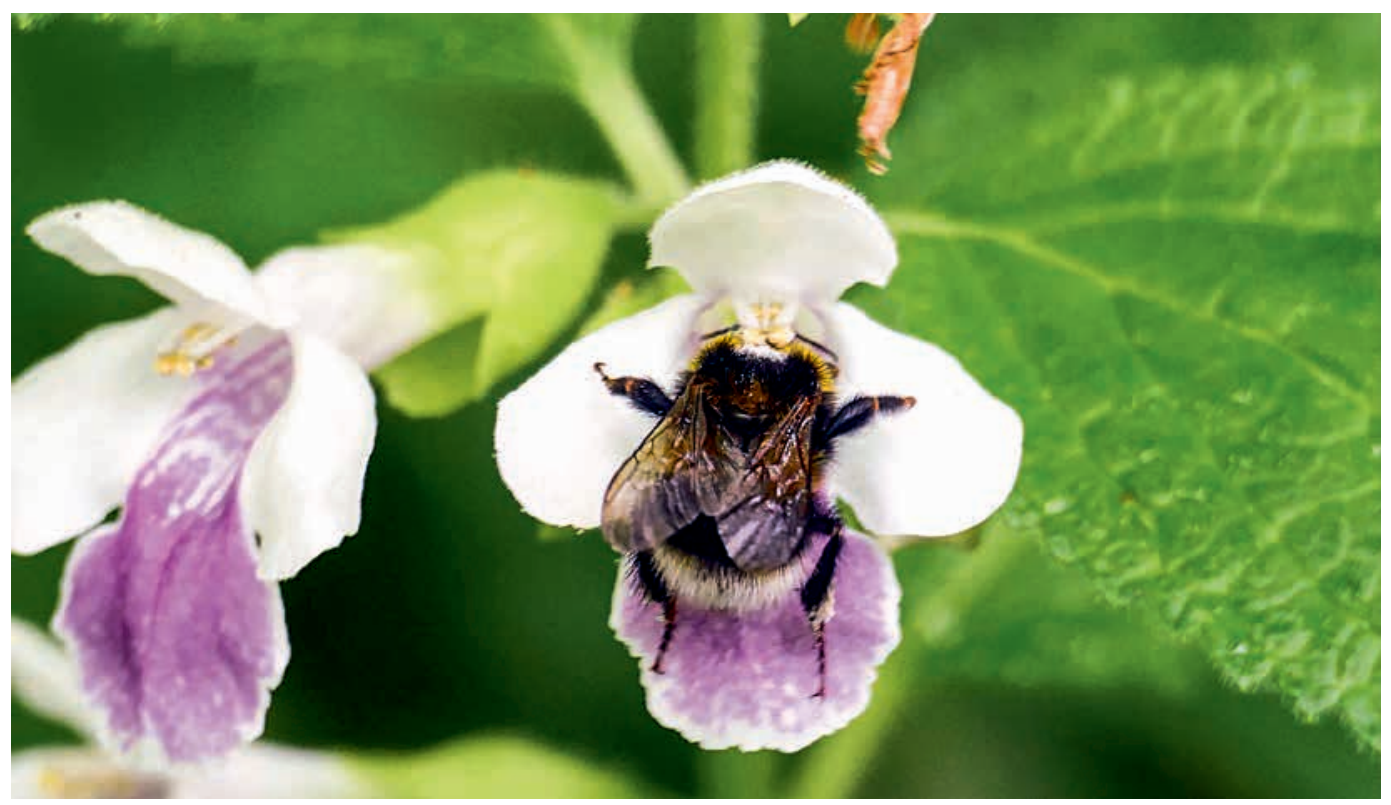

Abb. 11: Die Pollensäcke des Immenblattes berühren den Rücken der Hummel, den sie dabei mit Pollen bepudern. (Foto: H. Steinecke)

\section{Melittis melissophyllum (Immenblatt, Lamia- ceae, Blumentyp: Lippenblume)}

Das Immenblatt ist eine typische Lippenblume, wobei die Unterlippe meist auffällig gefärbt ist. Als Nahrung werden sowohl Nektar am Blütengrund als auch Pollen angeboten. Wichtigste Bestäuber sind Hummeln und Schmetterlinge. Sie gelangen mit ihren relativ langen Rüsseln ins Blüteninnere. Die Hummel zwängt sich in die Blüten hinein und wird dabei am Kopf oder Rücken mit Blütenstaub betupft.

\section{Salvia sclarea (Muskateller-Salbei, Lamiaceae, Blumentyp: Lippenblume)}

Eine große Schauwirkung haben die hellblau bis rosa gefärbten Tragblätter der Scheinquirle. Die Blüten sind deutlich in eine blaue Oberlippe und eine weiße Unterlippe gegliedert. Am Grunde der Blüten wird Nektar angeboten, der besonders gern von den großen Holzbienen, die genau in die großen Blüten passen, gesammelt wird. An der Basis der Blüte befindet sich eine Platte, mit der die beiden Staubfäden der zwei Staubblätter in der Blüte verankert sind. Während der Nektarsuche drückt das Insekt die Platte nach hinten und wie zwei Hebelarme werden die Staubfäden nach

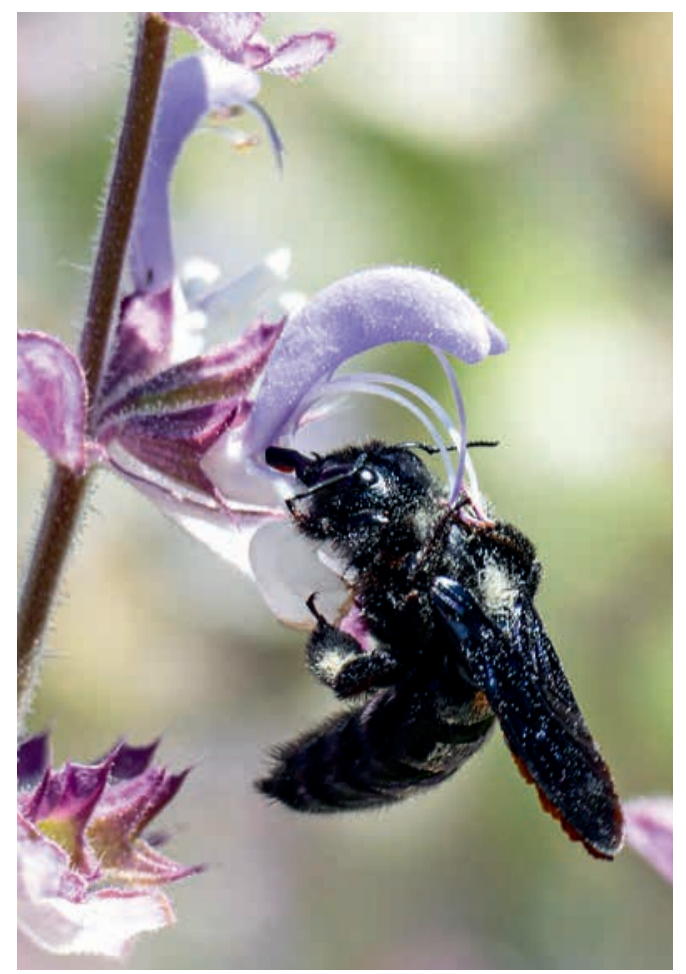

Abb. 12: Eine Große Holzbiene (Xylocopa violacea) hat am Muskateller-Salbei den Hebelmechanismus ausgelöst. (Foto: H. STEINECKe) 


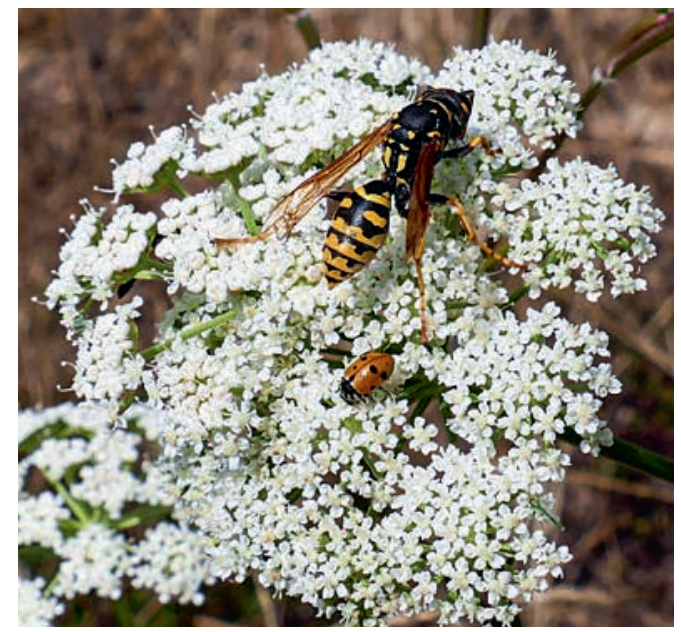

Abb. 13: Gallische Feldwespe (Pollistes dominula) und Variabler Flach-Marienkäfer (Hippodamia variegata) auf einer Blütendolde des Steppenfenchels. (Foto: H. Steinecke)

unten geklappt, so dass der Blütenstaub aus den Pollensäcken auf dem Rücken des Tieres abgeladen wird (Hebelmechanismus).

\section{Seseli annuum (Steppenfenchel, Apiaceae, Blumentyp: Scheibenblume)}

Der Blütenstand ist eine Doppeldolde mit bis zu 40 Doldenstrahlen. Mit ihren vielen weißen bis zart rosa gefärbten Blüten wirkt die Dolde wie eine große Einzelblüte. Um den Fruchtknoten liegt ein Nektarium, das für alle Insekten frei zugänglichen Nektar absondert. Fliegen, Käfer und Hautflügler sind hier häufige Blütenbesucher und Bestäuber. Es kommt aber auch Selbstbestäubung vor.

\section{Dank}

Unser Dank für Anregungen zur Anlage eines Bestäuberbeetes und zur Artenauswahl geht an verschiedenste Botanische Gärten, so auch nach Aachen, Berlin, Bremen, Düsseldorf, Erlangen, Hannover, Köln, Konstanz, Tübingen und Wien sowie an Sonja Lütgehetmann und Ditmar BreimHORST. Hilfreich war auch die Broschüre „Fingerhut ruft Hummel“ (Lehnert \& Wöhrmann 1998).

\section{Literatur}

Lehnert, H. J. \& Wöhrmann, F. (Hrsg.) 1998: Fingerhut ruft Hummel. Blütenökologie an Botanischen Gärten. Ergeb- nisse der 12. Arbeitstagung Pädagogischer Mitarbeiterinnen und Mitarbeiter im Verband Botanischer Gärten e. V. in Greifswald im Juni 1997. - Osnabrück.

Steinecke, H., Schmidt, M., Becker, A., Hillenbrand, J. \& Vaupel, B. 2021: Tropische Falter im neuen Blüten- und Schmetterlingshaus des Palmengartens. - Palmengarten 85: 3-19

Weber. A. \& Steinecke, H. 2016: Die großen wissenschaftlichen Leistungen von Stefan Vogel (1925 - 2015). Teil 1. Die Wiederentdeckung der Bestäubungssyndrome. - Palmengarten 80: 51-60.

Weber, A. \& Steinecke. H. 2016: Die großen wissenschaftlichen Leistungen von Stefan Vogel (1925-2015). Teil 2: Fledermausblumen und Blumenfledermäuse. - Palmengarten 80: $136-145$

Weber, A. \& Steinecke, H. 2017: Die großen wissenschaftlichen Leistungen von Stefan Vogel (1925-2015). Teil 3. Blütendüfte und ihre Bildung in den Osmophoren - Palmengarten 81: 69-77

Weber, A. \& Gerlach. G. 2017: Die großen wissenschaftlichen Leistungen von Stefan Vogel (1925-2015). Teil 4. Die Parfümblumen und ihre prächtigen Bestäuber. - Palmengarten 81: 133-148.

Weber, A., Gerlach, G. \& Dötterl, S. 2018: Die großen wissenschaftlichen Leistungen von STEFAN Vogel (19252015) Teil 5a: Öl statt Nektar - die Ölblumen (Allgemeine Aspekte). - Palmengarten 82(1): 49-61.

Weber, A., Gerlach, G. \& Dötterl, S. 2018: Die großen wissenschaftlichen Leistungen von Stefan Vogel (19252015). Teil 5b. Öl statt Nektar - Die Ölblumen (dikotyle Vertreter). - Palmengarten 82(2): 48-65.

Weber, A., Gerlach, G. \& Schaefer, H. 2019: Die großen wissenschaftlichen Leistungen von STEFan VogeL (19252015). Teil 5c. Öl statt Nektar - die Ölblumen (monokotyle Vertreter und abschöießende Bemerkungen) - Palmengarten 83: $41-53$.

\section{Internetseite}

iNaturalist (https://www.inaturalist.org/projects/tiere-undpilze-in-frankfurts-botanischen-garten).

\section{Anschriften der Autoren und der Autorin}

Andreas König, Palmengarten - Botanischer Garten, Siesmayerstraße 61, 60323 Frankfurt am Main, E-Mail: andreas.koenig@stadt-frankfut.de

Tномаs Moos, Palmengarten - Botanischer Garten, Siesmayerstraße 61, 60323 Frankfurt am Main, E-Mail: thomas.moos.Amt78@stadt-frankfut.de

Dr. Hilke Steinecke, Palmengarten, Siesmayerstraße 61, 60323 Frankfurt am Main,

E-Mail: hilke.steinecke@stadt-frankfut.de 